\title{
All-Order Amplitudes at any Multiplicity in the Multi-Regge Limit
}

\author{
V. Del Duca $\odot,{ }^{1,2}$ S. Druc, ${ }^{3}$ J. M. Drummond, ${ }^{3}$ C. Duhr, ${ }^{4}$ F. Dulat, ${ }^{5}$ R. Marzucca $\odot,{ }^{6}$ \\ G. Papathanasiou, ${ }^{7}$ and B. Verbeek ${ }^{8}$ \\ ${ }^{1}$ Institute for Theoretical Physics, ETH Zürich, 8093 Zürich, Switzerland \\ ${ }^{2}$ INFN, Laboratori Nazionali di Frascati, 00044 Frascati (RM), Italy \\ ${ }^{3}$ School of Physics and Astronomy, University of Southampton, Highfield SO17 1BJ, United Kingdom \\ ${ }^{4}$ Theoretical Physics Department, CERN, CH-1211 Geneva 23, Switzerland \\ ${ }^{5}$ SLAC National Accelerator Laboratory, Stanford University, Stanford, California 94309, USA \\ ${ }^{6}$ IPPP, Department of Physics, Durham University, Durham DH1 3LE, United Kingdom \\ ${ }^{7}$ DESY Theory Group, DESY Hamburg, Notkestraße 85, D-22607 Hamburg, Germany \\ ${ }^{8}$ Department of Physics and Astronomy, Uppsala University, 75108 Uppsala, Sweden
}

(Received 17 January 2020; accepted 25 March 2020; published 20 April 2020)

\begin{abstract}
We propose an all-loop expression for scattering amplitudes in planar $\mathcal{N}=4$ super Yang-Mills theory in multi-Regge kinematics valid for all multiplicities, all helicity configurations, and arbitrary logarithmic accuracy. Our expression is arrived at from comparing explicit perturbative results with general expectations from the integrable structure of a closely related collinear limit. A crucial ingredient of the analysis is an allorder extension for the central emission vertex that we recently computed at next-to-leading logarithmic accuracy. As an application, we use our all-order formula to prove that all amplitudes in this theory in multiRegge kinematics are single-valued multiple polylogarithms of uniform transcendental weight.
\end{abstract}

DOI: $10.1103 /$ PhysRevLett.124.161602

Recent years have seen tremendous progress in our understanding of multiloop, multileg scattering amplitudes in planar $\mathcal{N}=4$ super Yang-Mills (SYM) theory. Its $S$ matrix exhibits a hidden dual conformal (DC) symmetry [1], which closes with the ordinary conformal symmetry into a Yangian algebra [2].

The DC symmetry is broken by infrared (IR) divergences. Such divergences are universal and independent of the hard scattering process and it is possible to construct DC-invariant functions by considering ratios where all IR divergences cancel. We denote by $\mathcal{R}_{N}$ the IR-finite ratio of the $N$-point color-ordered amplitude and the Bern-DixonSmirnov (BDS) ansatz [3], defined (loosely) as the exponential of the one-loop amplitude multiplied by the cusp anomalous dimension $\Gamma_{\text {cusp }}$ [4]. DC invariance dictates that $\mathcal{R}_{N}$ only depends on $3 N-15$ independent cross ratios. In particular, $\mathcal{R}_{N}$ is trivial for $N \leq 5$ [5], and is known analytically in general kinematics for $N=6$ through seven loops [6-17] and for $N=7$ through four loops [18-22], at the level of the symbol [8].

Explicit data for small $N$ reveal that the perturbative expansion of $\mathcal{R}_{N}$ can often be expressed in terms of a class of iterated integrals known as "multiple polylogarithms"

Published by the American Physical Society under the terms of the Creative Commons Attribution 4.0 International license. Further distribution of this work must maintain attribution to the author(s) and the published article's title, journal citation, and DOI. Funded by SCOAP.
(MPLs) [23]. Moreover, only MPLs of (transcendental) weight $2 L$ contribute to an $L$-loop amplitude, where weight is the number of iterated integrations.

The mathematical beauty and simplicity of the available perturbative results hint at some deeper structure governing amplitudes in planar $\mathcal{N}=4$ SYM theory. This is corroborated by the fact that infinite-dimensional symmetries, like the Yangian symmetry of $\mathcal{N}=4 \mathrm{SYM}$, are a hallmark of integrability. One should then be able to compute $\mathcal{R}_{N}$ at any value of the coupling. A major step in this direction was taken in [24-28], where it was argued that amplitudes (or their dual Wilson loops [29-33]) can be computed through an integrable flux-tube picture. The dream of computing amplitudes analytically at any value of the coupling constant $g^{2}$, or at least at any order in perturbation theory, has not yet been achieved.

Here we present for the first time a way to compute scattering amplitudes in planar $\mathcal{N}=4 \mathrm{SYM}$ to any order in the coupling, for any helicity configuration and any number of external legs, albeit in the simplified kinematic setup of multi-Regge kinematics (MRK), where the produced particles are strongly ordered in rapidity and have comparable transverse momenta. While in Euclidean kinematics the ratios $\mathcal{R}_{N}$ become trivial in the limit [34-39], they develop a nontrivial kinematic dependence when some of the energies of the produced gluons are analytically continued to negative values [35,37]. Here we focus on the situation where all the centrally produced gluons have a negative energy, and we propose a formula for any amplitude in MRK in this theory. 


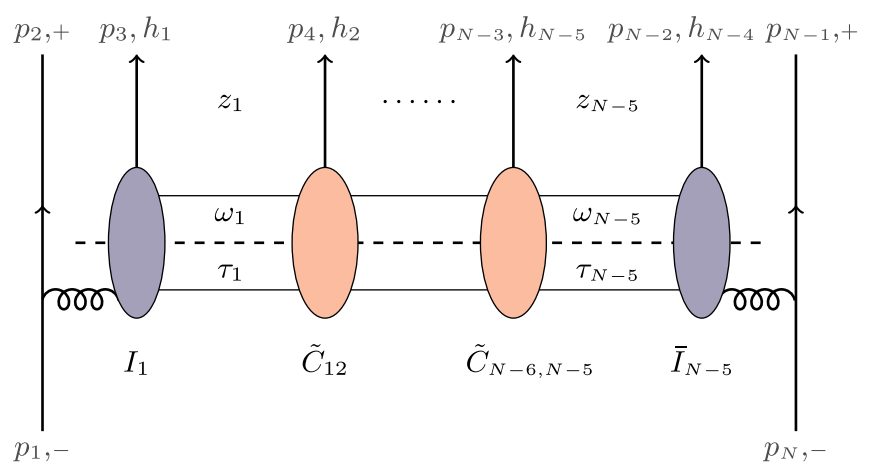

FIG. 1. Fourier-Mellin factorization of $2 \rightarrow N-2$ gluon amplitude in multi-Regge kinematics.

The N-particle dispersion integral.-In MRK, a subset of $N-5$ cross ratios, denoted by $\tau_{i}$, approach zero. $\mathcal{R}_{N}$ can then be expressed at each order as a polynomial in large $\operatorname{logarithms} \log \tau_{i}$, multiplied by functions of the $2 N-10$ remaining real degrees of freedom. The latter are conveniently described by $N-5$ complex variables $z_{i}$ (see [40] and references therein for these standard conventions). We conjecture that, to all orders, $\mathcal{R}_{N}$ can be written as a Fourier-Mellin (FM) integral with a factorized form, as also depicted in Fig. 1,

$$
\begin{aligned}
\frac{\mathcal{R}_{N} e^{i \Gamma \delta}}{2 \pi i}= & \prod_{r=1}^{N-5}\left[\sum_{n_{r}}\left(\frac{z_{r}}{\bar{z}_{r}}\right)^{n_{r} / 2} \int_{\mathcal{C}} \frac{d \nu_{r}}{2 \pi} \frac{\left|z_{r}\right|^{2 i \nu_{r}} \tilde{\Phi}_{r}}{\left(-\tau_{r}+i 0\right)^{\omega_{r}}}\right] \\
& \times I_{1}^{h_{1}} \tilde{C}_{12}^{h_{2}} \ldots \tilde{C}_{N-6, N-5}^{h_{N-5}} \bar{I}_{N-5}^{h_{N-4}} .
\end{aligned}
$$

Equation (1) extends similar formulas in the literature for restricted subsets of amplitudes at leading logarithmic accuracy (LLA) and beyond [37,40-46] (see also [47] for an application). The ratio $\mathcal{R}_{N}$ depends on the helicities $h_{r}$ of all centrally produced particles. The building blocks of the integrand $\omega_{r}, \tilde{\Phi}_{r}, I_{r}$, and $\tilde{C}_{r, r+1}^{h_{r+1}}$ are known as the Balitsky-Fadin-Kuraev-Lipatov (BFKL) eigenvalue, impact factor product, helicity flip kernel, and (rescaled) central emission block (see aforementioned references and references therein). They are functions of the FM variables $\left(\nu_{r}, n_{r}\right)$, whose precise form will be presented below, and we use a shorthand notation $\omega_{r}=\omega\left(\nu_{r}, n_{r}\right)$ and $\tilde{C}_{r, r+1}^{h_{r+1}}=\tilde{C}^{h_{r+1}}\left(\nu_{r}, n_{r}, \nu_{r+1}, n_{r+1}\right)$, etc. The phase $e^{i \Gamma \delta}$, where $\Gamma \equiv \Gamma_{\text {cusp }} / 4$, captures terms in the BDS ansatz that do not vanish after analytic continuation in MRK [48].

In the limit where one of the centrally produced gluons becomes soft, $\mathcal{R}_{N}$ should reduce to $\mathcal{R}_{N-1}$. Provided that the building blocks have at most simple poles on the integration axis, this then dictates that the contour $\mathcal{C}$ must take the form shown in Fig. 2 and implies the following exact bootstrap conditions $[46,50]$ :

$$
\omega( \pm \pi \Gamma, 0)=0, \quad \operatorname{Res}_{\nu= \pm \pi \Gamma}[\tilde{\Phi}(\nu, 0)]= \pm \frac{1}{2 \pi},
$$

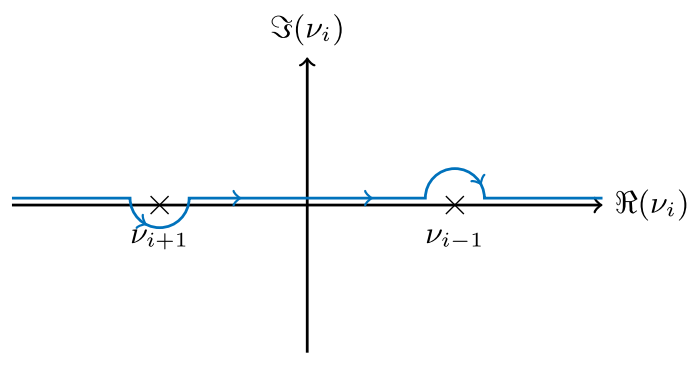

FIG. 2. Contour of integration $\mathcal{C}$ for the integral (1), with $\nu_{N-4}=-\pi \Gamma, \nu_{0}=\pi \Gamma$ corresponding to the boundary cases.

$$
\begin{gathered}
\tilde{C}^{h}\left(\pi \Gamma, 0, \nu_{2}, n_{2}\right)=2 \pi i I^{h}\left(\nu_{2}, n_{2}\right), \\
\tilde{C}^{h}\left(\nu_{1}, n_{1},-\pi \Gamma, 0\right)=-2 \pi i \bar{I}^{h}\left(\nu_{1}, n_{1}\right), \\
\operatorname{Res}_{\nu_{1}=\nu_{2}} \tilde{C}^{h}\left(\nu_{1}, n_{2}, \nu_{2}, n_{2}\right)=\frac{-i(-1)^{n_{2}} e^{i \pi \omega\left(\nu_{2}, n_{2}\right)}}{\tilde{\Phi}\left(\nu_{2}, n_{2}\right)}, \\
\tilde{C}^{h}\left(-\pi \Gamma, 0, \nu_{2}, n_{2}\right)=\tilde{C}^{h}\left(\nu_{1}, n_{1}, \pi \Gamma, 0\right)=0 .
\end{gathered}
$$

Let us now proceed to fully specify the integral (1), by providing explicit expressions for its building blocks. The BFKL eigenvalue $\omega_{r}$, impact factor product $\tilde{\Phi}_{r}$, and helicity flip kernel $I_{r}$ have already been determined to all loops [28], by means of an analytic continuation from the collinear limit. The latter limit is also described by a dispersion integral very similar to (1), whose building blocks are governed by an integrable flux tube and may thus be computed at finite coupling within the pentagon operator product expansion (OPE) [24-27] approach. Then, the authors of [28] were able to connect the multi-Regge and collinear integrands by analytically continuing in the integration variable and, in particular, obtain $\omega_{r}, \tilde{\Phi}_{r}$, and $I_{r}$ from their OPE counterparts, the gluonic excitation energy, measure, and next-to maximally helicity violating (NMHV) impact factor respectively. A feature of this analysis is that at finite coupling it is more natural to use rapidities $u_{r}$ rather than $\nu_{r}$ as integration variables, giving rise to the following implicit all-loop dispersion relation:

$\nu_{r}=u_{r}-2 g(\mathbb{Q} \cdot \mathbb{M} \cdot \tilde{\kappa})_{1}, \quad \omega_{r}=-4 g(\mathbb{Q} \cdot \mathbb{M} \cdot \kappa)_{1}$.

The sources $\kappa$ and $\tilde{\kappa}$ are infinite-dimensional vectors and are described explicitly in the Supplemental Material [51] along with the matrices $\mathbb{Q}$ and $\mathbb{M}$, which essentially encode the Beisert-Eden-Staudacher kernel [4,52]. The subscript 1 in (7) means the first component of the vector.

Central emission vertex.-The only quantity in (1) only known at leading order (LO) [43] and next-to-LO [46] is the central emission vertex $C_{r, r+1}^{ \pm}$. A main result of this 
Letter is a conjecture for $C_{r, r+1}^{ \pm}$to all orders in the coupling, as we now move on to describe. We focus on the vertex for the emission of a positive helicity gluon. The case of negative helicity is then recovered from the helicity flip kernel [53],

$$
\tilde{C}_{r, r+1}^{-}=\tilde{C}_{r, r+1}^{+} \bar{I}_{r}^{-} I_{r+1}^{-} .
$$

Our analysis parallels that of [28] for $N=6$. We assume that also for $N=7$, the dispersion integral (1) can be obtained by analytically continuing the contribution of gluon excitations to the pentagon OPE through the branch cut at $u_{r}=-i n_{r} / 2 \pm 2 g$ in the rapidity plane. It follows that the central emission vertex is the analytic continuation of the new OPE building block appearing at this multiplicity, known as the "gluon pentagon transition" [27]. Performing the analytic continuation in full generality is quite complicated, but we are able to present a conjectural all-orders form for the central emission vertex by continuing certain factors of the pentagon transition and fixing the remaining proportionality coefficient by consistency with known perturbative data in MRK. More precisely, our conjecture reads

$\tilde{C}_{12}^{+}=\frac{\tilde{C}_{12}^{(0)}}{g^{2}} k_{12} Z_{12} \exp \left(f_{12}-f_{\tilde{1} \tilde{2}}-i f_{\tilde{1} 2}+i f_{1 \tilde{2}}-A\right)$.

Here $\tilde{C}_{12}^{(0)}$ denotes the LO central emission vertex of Ref. [43], with the $\nu_{r}$ replaced with the rapidities $u_{r}$,

$\tilde{C}_{12}^{(0)}=\frac{\Gamma\left(1-i u_{1}-\frac{n_{1}}{2}\right) \Gamma\left(1+i u_{2}+\frac{n_{2}}{2}\right) \Gamma\left(i u_{1}-i u_{2}-\frac{n_{1}-n_{2}}{2}\right)}{\Gamma\left(i u_{1}-\frac{n_{1}}{2}\right) \Gamma\left(-i u_{2}+\frac{n_{2}}{2}\right) \Gamma\left(1-i u_{1}+i u_{2}-\frac{n_{1}-n_{2}}{2}\right)}$.

The exponential factor and $Z_{12}$ in (9) are obtained by analytically continuing the corresponding functions appearing in the pentagon transition [54]. The functions $f_{r s}$ are given by

$$
f_{r s}=4 \kappa\left(u_{r}, n_{r}\right) \cdot \mathbb{Q} \cdot \mathbb{M} \cdot \kappa\left(u_{s}, n_{s}\right),
$$

similarly, $f_{\tilde{r} s}\left(f_{\tilde{r} \tilde{s}}\right)$ for $\kappa_{r} \rightarrow \tilde{\kappa}_{r}$ (and $\left.\kappa_{s} \rightarrow \tilde{\kappa}_{s}\right)$, in terms of the same sources $\kappa, \tilde{\kappa}$ appearing in [51]. The constant $A$ is given by

$$
A=2 \int_{0}^{\infty} \frac{d t}{t} \frac{1-J_{0}(2 g t)^{2}}{e^{t}-1}-\pi^{2} \Gamma .
$$

For $Z_{12}$ we have

$$
Z_{12}=\sqrt{\frac{\left(x_{1}^{-} x_{2}^{-}-g^{2}\right)\left(x_{1}^{+} x_{2}^{+}-g^{2}\right)}{\left(x_{1}^{+} x_{2}^{-}-g^{2}\right)\left(x_{1}^{-} x_{2}^{+}-g^{2}\right)}},
$$

where we introduce the Zhukowski variables

$x_{r}^{ \pm}=x\left(u_{r} \pm i \frac{n_{r}}{2}\right), \quad x\left(u_{r}\right)=\frac{1}{2}\left(u_{r}+\sqrt{u_{r}^{2}-4 g^{2}}\right)$.

The quantity $k_{12}$ in (9) collects all the factors we have not addressed so far, and is a priori unknown. Nevertheless, it is constrained by the exact bootstrap condition (5) to be free of poles at $u_{r}=u_{s}$, and this condition also fixes the value of $k_{12}$ at $\left(u_{2}, n_{2}\right)=\left(u_{1}, n_{1}\right)$ to be

$$
\begin{aligned}
k_{12 \mid\left(u_{2}, n_{2}\right)=\left(u_{1}, n_{1}\right)} & =\frac{x^{+} x^{-}}{u_{1}^{2}+\frac{n_{1}^{2}}{4}} e^{i \pi \omega_{1}} \\
& =e^{2 \int_{0}^{\infty}(d t / t)\left[1-J_{0}(2 g t)\right] \cos \left(u_{1} t\right) e^{-\left(n_{1} / 2\right) t}+i \pi \omega_{1}} .
\end{aligned}
$$

There could be many functions $k_{12}$ that satisfy (15), but there is a particularly simple solution where $k_{12}$ takes a factorized form,

$$
k_{12}=k_{1} \breve{k}_{2}, \quad \check{k}(u, n)=k(-u,-n) .
$$

This form is motivated by the fact that it reproduces the perturbative expansion of the same quantity to three loops, extracted from the corresponding seven-particle maximally helicity violating (MHV) amplitude [20] with the method described in [46]. We conjecture that this minimal form persists to all orders in perturbation theory. Inserting the factorized form into (15), we find

$$
k_{1}=\sqrt{\frac{x^{+} x^{-}}{u_{1}^{2}+\frac{n_{1}^{2}}{4}}} e^{(i / 2) \pi \omega_{1}} k_{o 1}, \quad \check{k}_{o 1}=k_{o 1}^{-1} .
$$

The remaining freedom $k_{o 1}$ can be determined by solving the exact bootstrap condition (4) order by order in perturbation theory. We observe empirically that the perturbative expansion of $k_{o 1}$ is consistent with an exponential form for $k_{o 1}$ very reminiscent of (15),

$$
k_{o 1}=e^{i \int_{0}^{\infty}(d t / t) \frac{\left(J_{0}(2 g t)-1\right)\left(e^{t}+1\right)}{\left(e^{t}-1\right)} \sin \left(u_{1} t\right) e^{-\left(n_{1} / 2\right) t}+\pi\left(u_{1}-\nu_{1}\right)} .
$$

This concludes our conjecture for the all-order structure of $\mathcal{R}_{N}$ in MRK. In fact, the dispersion integral (1) is valid also at finite coupling, and so is the central emission block (9), for all integer angular momenta $n_{r}$ different from zero. As noted in [28], a subtlety that appears when $n_{r}=0$ is that one needs two sheets in the rapidity $u_{r}$ in order to cover the entire real $\nu_{r}$ line, with the expressions (10)-(18) only covering the interval $\left|\nu_{r}\right| \geq \tilde{\nu}_{r}=\nu\left(u_{r}=2 g\right)$ (this is not an issue at weak coupling, where we can express all building blocks as functions of $\nu_{r}$ directly). Covering also the $\left|\nu_{r}\right|<\tilde{\nu}_{r}$ interval would additionally serve as a starting point for analyzing the strong-coupling limit and making contact with the string-theoretic description of the same regime [55]. 
The perturbative expansion of all quantities entering (1) is simple to obtain $[25,27,56]$, since at fixed order only a finite number of components of the vectors $\kappa, \tilde{\kappa}$ contribute. The coefficients of the perturbative expansion take a very special form; the ratio to their leading-order contribution is always a polynomial in the following "FM building blocks," first introduced in [46,57],

$$
\begin{aligned}
V_{i}= & \frac{i \nu_{i}}{\nu_{i}^{2}+\frac{n_{i}^{2}}{4}}, \quad N_{i}=\frac{n_{i}}{\nu_{i}^{2}+\frac{n_{i}^{2}}{4}}, \quad D_{i}=-i \frac{\partial}{\partial \nu_{i}}, \\
E_{i}= & \psi\left(1+i \nu_{i}+\frac{\left|n_{i}\right|}{2}\right)+\psi\left(1-i \nu_{i}+\frac{\left|n_{i}\right|}{2}\right) \\
& -2 \psi(1)-\frac{1}{2} \frac{\left|n_{i}\right|}{\nu_{i}^{2}+\frac{n_{i}^{2}}{4}}, \\
M_{i j}= & \psi\left(i \nu_{i j}-\frac{n_{i j}}{2}\right)+\psi\left(1-i \nu_{i j}-\frac{n_{i j}}{2}\right)-2 \psi(1),
\end{aligned}
$$

where $\nu_{i j}=\nu_{i}-\nu_{j}, n_{i j}=n_{i}-n_{j}$, and $\psi(z)=\partial_{z} \ln \Gamma(z)$ is the digamma function.

We implement the general expansion of $\tilde{C}_{12}^{+}$and provide explicit results through five loops, as ancilliary files in the arXiv preprint version of this Letter. As independent checks, we have verified that by inserting it into the dispersion integral (1) and evaluating, we find perfect agreement for the imaginary part of the four-loop seven-particle MHV symbol [21], as well as for the twoloop MHV amplitude at any multiplicity [46,58]. More details on the integral evaluation step are provided in the next section.

Analytic loop amplitudes in MRK.-In this section we provide the last ingredient needed to compute amplitudes from the dispersive representation in Eq. (1), and we discuss how the integrals can be efficiently performed in terms of the relevant class of functions in the limit, known as "singlevalued MPLs" (SVMPLs) [40,59,60]. As an application, we will give for the first time a proof of the principle of uniform and maximal transcendentality in MRK: An $L$-loop gluon amplitude in MRK in planar $\mathcal{N}=4$ SYM is a linear combination of products of $\log \tau_{i}$, SVMPLs, zeta values, and powers of $2 \pi i$ of uniform weight $2 L$, for any helicity configuration and any number of legs.

The proof is constructive, thereby providing an important algorithm to compute any scattering amplitude in MRK order by order in the coupling, as we now sketch. For $N=6$ gluons, similar proofs for the relevant classes of functions in the collinear and LLA multi-Regge limit have appeared in $[57,61,62]$ and $[63,64]$, respectively (see also [40] for an extension of the latter to any $N$ ).

We start by noting that at order $\mathcal{O}\left(g^{2}\right)$, the MHV amplitude will be the $(N-5)$-fold FM transform of the "vacuum ladder,"

$$
\varpi=\prod_{r=1}^{N-5} \frac{1}{\nu_{r}^{2}+\frac{n_{r}^{2}}{4}} \prod_{r=1}^{N-6} \tilde{C}_{r, r+1}^{(0)} .
$$

Letting $\mathcal{F}\left[X_{r}\right]$ denote the FM transform of $X_{r}$, we have, in particular, that $\mathcal{F}[\varpi]=\delta /(4 \pi)$, with $\delta$ as in (1) being of uniform weight 1 .

At higher loops, the integrand will be a product of (20) with sums of polynomials of the FM building blocks (19). If we assign weight 1 to them, and given that the polynomial coefficients are $\mathbb{Q}$-linear combinations of Riemann zeta values $\zeta_{n}=\zeta(n)$, whose weight is $n$, then we observe that these polynomials have uniform transcendental weight. In other words, we see that the all-order formulas obtained from integrability imply the principle of uniform and maximal transcendentality in FM space.

To go to momentum space, we then make use of the FM transform's property to map products to convolutions,

$$
\mathcal{F}[f g]=\mathcal{F}[f] * \mathcal{F}[g],
$$

where

$$
(F * G)(z)=\int \frac{d^{2} w}{|w|^{2}} F(w) G\left(\frac{z}{w}\right) .
$$

Every higher-loop amplitude in MRK can thus be built iteratively by convolving the vacuum ladder (20) with a finite number of FM building blocks (19). While the evaluation of the convolution integral seems a daunting task, it was shown in [65] (see also [40]) that, in the case where the integrand only involves rational functions and SVMPLs, the integral can easily be evaluated in terms of residues.

The proof now proceeds by induction: Assume we have a pure linear combination of SVMPLs of uniform weight. We will show that convolution with any FM building block raises the weight by one and preserves purity. This justifies our assignment of weight 1 to the building blocks and implies that all MHV amplitudes in MRK satisfy the principle of uniform and maximal transcendentality.

More concretely, assume that $f(z)$ is a pure linear combination of SVMPLs of uniform weight $n$ and let

$$
\mathcal{K}(z)=|z|^{2} \sum_{i, j} \frac{a_{i j}}{\left(z-\alpha_{i}\right)\left(\bar{z}-\beta_{j}\right)},
$$

with $a_{i j}, \alpha_{i}, \beta_{j} \in \mathbb{Q}$. One can show using Stokes's theorem [65] that $(f * \mathcal{K})(z)$ is again pure and has uniform weight $n+1$. The FM transform of the building blocks $E_{r}, N_{r}, V_{r}$ match the form in $(23)[40,46,47]$

$$
\begin{gathered}
\mathcal{F}\left[E_{r}\right]=-\frac{z_{r}+\bar{z}_{r}}{2\left|1-z_{r}\right|^{2}}, \\
\mathcal{F}\left[V_{r}\right]=\frac{2-z_{r}-\bar{z}_{r}}{2\left|1-z_{r}\right|^{2}}, \\
\mathcal{F}\left[N_{r}\right]=\frac{z_{r}-\bar{z}_{r}}{\left|1-z_{r}\right|^{2}} .
\end{gathered}
$$


Hence, they raise the weight of the function they are convolved with by one. We may similarly show that the same holds true for the derivative $D_{r}$, by using integration by parts to let it act on the factor $\left|z_{r}\right|^{2 \nu_{r}}$ in the definition of the FM transform,

$$
\mathcal{F}\left[D_{r} X_{r}\right]=-\log \left|z_{r}\right|^{2} \mathcal{F}\left[X_{r}\right]
$$

Finally, let us note that the FM building block $M_{r s}$ obeys

$$
\begin{aligned}
M_{r s} & =D_{r} \log \left(\tilde{C}_{r s}^{(0)}\right)+E_{r}+V_{r} \\
& =-D_{s} \log \left(\tilde{C}_{r s}^{(0)}\right)+E_{s}-V_{s} .
\end{aligned}
$$

This allows us to shift occurrences of $M_{r s}$ in its FM transform with the vacuum ladder to either end,

$$
\begin{aligned}
\mathcal{F}\left[\varpi M_{r r+1}\right] & =\mathcal{F}\left[\varpi M_{r-1 r}\right]+\mathcal{F}\left[D_{r} \varpi\right], \\
\mathcal{F}\left[\varpi M_{12}\right] & =\mathcal{F}\left[\varpi E_{1}\right]-\mathcal{F}\left[\varpi V_{1}\right]+\mathcal{F}\left[D_{1} \varpi\right],
\end{aligned}
$$

and in this manner replace it by a combination of $E, V, D$. Hence, $M_{r s}$ raises the weight of the integral by one as well. Finally, our proof may be immediately extended to nonMHV amplitudes as well. The latter can be obtained by convoluting MHV amplitudes with the helicity flip kernel $I^{-}$, and the only difference is that at LO the latter does not raise the weight and it does not preserve the purity of the function [40]. We therefore conclude that non-MHV amplitudes have the same weight as their MHV counterparts, but are no longer pure functions.

Conclusions. - We have presented a dispersion integral for all gluon amplitudes of arbitrary multiplicity and helicity configurations in MRK. By combining our results with [40], we obtain an efficient algorithm to evaluate any scattering amplitude in MRK, for any number of loops or legs, and for arbitrary helicity configurations.

We believe that our results, while complete for the sector of planar $\mathcal{N}=4$ super Yang-Mills theory that we have studied, should serve as the basis for many future generalizations in various directions. First, it should be straightforward to include the fermions and scalars into our expression or to consider more general Mandelstam regions $[49,58,66,67]$. We believe that a similar structure will survive for general gauge theories, at least in the planar limit, though the details will differ because, in general, dual conformal symmetry is broken. It would be very interesting to understand how the form of the amplitude generalizes beyond the planar limit.

We would like to thank Jochen Bartels and Benjamin Basso for comments on the manuscript. This work was supported in part by the ERC Consolidator Grant No. 648630 IQFT and the ERC Starting Grants No. 637019 MathAm and
No. 804286 UNISCAMP, as well as the U.S. Department of Energy (DOE) under Award No. DE-AC02-76SF00515.

[1] J. M. Drummond, J. Henn, G. P. Korchemsky, and E. Sokatchev, Nucl. Phys. B828, 317 (2010).

[2] J. M. Drummond, J. M. Henn, and J. Plefka, J. High Energy Phys. 05 (2009) 046.

[3] Z. Bern, L. J. Dixon, and V. A. Smirnov, Phys. Rev. D 72, 085001 (2005).

[4] N. Beisert, B. Eden, and M. Staudacher, J. Stat. Mech. (2007) P01021.

[5] J. M. Drummond, J. Henn, G. P. Korchemsky, and E. Sokatchev, Nucl. Phys. B826, 337 (2010).

[6] V. Del Duca, C. Duhr, and V. A. Smirnov, J. High Energy Phys. 03 (2010) 099.

[7] V. Del Duca, C. Duhr, and V. A. Smirnov, J. High Energy Phys. 05 (2010) 084.

[8] A. B. Goncharov, M. Spradlin, C. Vergu, and A. Volovich, Phys. Rev. Lett. 105, 151605 (2010).

[9] L. J. Dixon, J. M. Drummond, and J. M. Henn, J. High Energy Phys. 11 (2011) 023.

[10] L. J. Dixon, J. M. Drummond, and J. M. Henn, J. High Energy Phys. 01 (2012) 024.

[11] L. J. Dixon, J. M. Drummond, M. von Hippel, and J. Pennington, J. High Energy Phys. 12 (2013) 049.

[12] L. J. Dixon, J. M. Drummond, C. Duhr, and J. Pennington, J. High Energy Phys. 06 (2014) 116.

[13] L. J. Dixon, J. M. Drummond, C. Duhr, M. von Hippel, and J. Pennington, Proc. Sci., LL2014 (2014) 077.

[14] L. J. Dixon and M. von Hippel, J. High Energy Phys. 10 (2014) 065.

[15] L. J. Dixon, M. von Hippel, and A. J. McLeod, J. High Energy Phys. 01 (2016) 053.

[16] S. Caron-Huot, L. J. Dixon, A. McLeod, and M. von Hippel, Phys. Rev. Lett. 117, 241601 (2016).

[17] S. Caron-Huot, L. J. Dixon, F. Dulat, M. von Hippel, A. J. McLeod, and G. Papathanasiou, J. High Energy Phys. 08 (2019) 016.

[18] J. Golden and M. Spradlin, J. High Energy Phys. 08 (2014) 154.

[19] J. Golden, M. F. Paulos, M. Spradlin, and A. Volovich, J. Phys. A 47, 474005 (2014).

[20] J. M. Drummond, G. Papathanasiou, and M. Spradlin, J. High Energy Phys. 03 (2015) 072.

[21] L. J. Dixon, J. Drummond, T. Harrington, A. J. McLeod, G. Papathanasiou, and M. Spradlin, J. High Energy Phys. 02 (2017) 137.

[22] J. Drummond, J. Foster, O. Gurdogan, and G. Papathanasiou, J. High Energy Phys. 03 (2019) 087.

[23] A. B. Goncharov, arXiv:math/0103059v4.

[24] B. Basso, A. Sever, and P. Vieira, Phys. Rev. Lett. 111, 091602 (2013).

[25] B. Basso, A. Sever, and P. Vieira, J. High Energy Phys. 01 (2014) 008 .

[26] B. Basso, A. Sever, and P. Vieira, J. High Energy Phys. 08 (2014) 085.

[27] B. Basso, A. Sever, and P. Vieira, J. High Energy Phys. 09 (2014) 149. 
[28] B. Basso, S. Caron-Huot, and A. Sever, J. High Energy Phys. 01 (2015) 027.

[29] L. F. Alday and J. M. Maldacena, J. High Energy Phys. 06 (2007) 064.

[30] J. M. Drummond, G. P. Korchemsky, and E. Sokatchev, Nucl. Phys. B795, 385 (2008).

[31] A. Brandhuber, P. Heslop, and G. Travaglini, Nucl. Phys. B794, 231 (2008).

[32] Z. Bern, L. J. Dixon, D. A. Kosower, R. Roiban, M. Spradlin, C. Vergu, and A. Volovich, Phys. Rev. D 78, 045007 (2008).

[33] J. M. Drummond, J. Henn, G. P. Korchemsky, and E. Sokatchev, Nucl. Phys. B815, 142 (2009).

[34] R. C. Brower, H. Nastase, H. J. Schnitzer, and C.-I. Tan, Nucl. Phys. B814, 293 (2009).

[35] J. Bartels, L. N. Lipatov, and A. Sabio Vera, Phys. Rev. D 80, 045002 (2009).

[36] V. Del Duca and E. W. N. Glover, J. High Energy Phys. 05 (2008) 056.

[37] J. Bartels, L. N. Lipatov, and A. S. Vera, Eur. Phys. J. C 65, 587 (2010).

[38] R. C. Brower, H. Nastase, H. J. Schnitzer, and C.-I. Tan, Nucl. Phys. B822, 301 (2009).

[39] V. Del Duca, C. Duhr, and E. W. N. Glover, J. High Energy Phys. 12 (2008) 097.

[40] V. Del Duca, S. Druc, J. Drummond, C. Duhr, F. Dulat, R. Marzucca, G. Papathanasiou, and B. Verbeek, J. High Energy Phys. 08 (2016) 152.

[41] L. N. Lipatov, Theor. Math. Phys. 170, 166 (2012).

[42] V. S. Fadin and L. N. Lipatov, Phys. Lett. B 706, 470 (2012).

[43] J. Bartels, A. Kormilitzin, L. N. Lipatov, and A. Prygarin, Phys. Rev. D 86, 065026 (2012).

[44] L. Lipatov, A. Prygarin, and H. J. Schnitzer, J. High Energy Phys. 01 (2013) 068.

[45] J. Bartels, A. Kormilitzin, and L. N. Lipatov, Phys. Rev. D 89, 065002 (2014).

[46] V. Del Duca, S. Druc, J. Drummond, C. Duhr, F. Dulat, R. Marzucca, G. Papathanasiou, and B. Verbeek, J. High Energy Phys. 06 (2018) 116.

[47] R. Marzucca and B. Verbeek, J. High Energy Phys. 07 (2019) 039.
[48] Explicitly $\delta(z)=\pi \log \left[\left(\left|\rho_{1}\right|^{2}\right) /\left(\left|1-\rho_{1}\right|^{2}\left|1-\rho_{N-5}\right|^{2}\right)\right]$, where the $\rho_{i}$ are defined in terms of the $z_{i}$ via $z_{i}=$ $\left\{\left[\left(1-\rho_{i+1}\right)\left(\rho_{i}-\rho_{i-1}\right)\right] /\left[\left(1-\rho_{i-1}\right)\left(\rho_{i}-\rho_{i+1}\right)\right]\right\} \quad$ together with $\rho_{0}=0$ and $\rho_{N-4}=\infty$ (see, e.g., Refs. [41,43,45,49]).

[49] V. Del Duca, C. Duhr, F. Dulat, and B. Penante, J. High Energy Phys. 01 (2019) 162.

[50] S. Caron-Huot, J. High Energy Phys. 05 (2015) 093.

[51] See Supplemental Material at http://link.aps.org/ supplemental/10.1103/PhysRevLett.124.161602 for definitions of the BFKL sources and BES kernel (as well as computer-readable files containing the weak-coupling expansion of the central emission vertex, and of all remaining BFKL building blocks).

[52] M. K. Benna, S. Benvenuti, I. R. Klebanov, and A. Scardicchio, Phys. Rev. Lett. 98, 131603 (2007).

[53] Explicitly the helicity flip kernels are $I_{r}^{+}=1$ and $I_{r}^{-}=$ $x_{r}^{-} / x_{r}^{+}$, where the Zhukowski variables $x_{r}^{ \pm}$are given in Eq. (14).

[54] The measure is similarly obtained and is given by $\left(d \nu_{r} / 2 \pi\right) \tilde{\Phi}_{r}=\left(d u_{r} / 2 \pi\right)\left\{\left[g^{2}\left(x_{r}^{+} x_{r}^{-}-g^{2}\right) \exp \left(A-f_{r r}+f_{\tilde{r} \tilde{r}}\right)\right] /\right.$ $\left.\left[x_{r}^{+} x_{r}^{-} \sqrt{\left(x_{r}^{+} x_{r}^{+}-g^{2}\right)\left(x_{r}^{-} x_{r}^{-}-g^{2}\right)}\right]\right\}$, see Ref. [28].

[55] J. Bartels, V. Schomerus, and M. Sprenger, J. High Energy Phys. 10 (2014) 67.

[56] J. M. Drummond and G. Papathanasiou, J. High Energy Phys. 02 (2016) 185.

[57] L. J. Dixon, C. Duhr, and J. Pennington, J. High Energy Phys. 10 (2012) 074.

[58] T. Bargheer, G. Papathanasiou, and V. Schomerus, J. High Energy Phys. 05 (2016) 012.

[59] F. C. S. Brown, http://www.ihes.fr/ brown/RHpaper5.pdf.

[60] F. C. S. Brown, arXiv:1512.06410.

[61] G. Papathanasiou, J. High Energy Phys. 11 (2013) 150.

[62] G. Papathanasiou, Int. J. Mod. Phys. A 29, 1450154 (2014).

[63] J. Pennington, J. High Energy Phys. 01 (2013) 059.

[64] J. Broedel and M. Sprenger, J. High Energy Phys. 05 (2016) 055.

[65] O. Schnetz, Commun. Num. Theor. Phys. 08, 589 (2014).

[66] T. Bargheer, J. High Energy Phys. 11 (2017) 077.

[67] T. Bargheer, V. Chestnov, and V. Schomerus, arXiv:1906 .00990 . 